\title{
Remote Opener: Breaking Barriers to Crystallization Using Remote Crystal Growth Screening and Imaging
}

\author{
S Bowman ${ }^{1}$ \\ ${ }^{1}$ Hauptman-Woodward Medical Research Institute, Buffalo, NY \\ sbowman@hwi.buffalo.edu
}

The vast majority of biomolecular structural information is derived from macromolecular X-ray crystallography methods, which serve as a foundation for structural biology and account for nearly $90 \%$ of the more than 165,000 biomolecular structures available in the PDB. Crystallography requires high-quality, well-diffracting crystals; coaxing biomolecules into crystalline form is a rate-limiting step in structure determination. Searching for conditions in which a biomolecule will crystallize often entails screening multiple different constructs against thousands of crystallization conditions, requiring large sample amounts and many person-hours in a typical laboratory set-up. In recent circumstances due to the COVID-19 pandemic, being physically in the laboratory for setting up crystallization screening has become even more difficult. The Crystallization Center at HWI has been in continuous operation as a crystallization resource for 20 years providing mail-in crystallization and remote access to crystal growth monitoring. These services have become even more critical in the face of restrictions due to COVID-19. The Crystallization Center is a high-throughput facility that provides expertise and access to state-ofthe-art instrumentation to facilitate efficient and cost-effective crystallization. We have extensive robotics for automated sample handling with very small sample volumes integrated with advanced imaging and a Formulatrix Rock Imager with SONICC for rapid detection of crystal growth. The current pipeline in the Crystallization Center screens for 1,536 conditions in one experimental plate and employs a robust imaging schedule, all of which is then accessible remotely. Here, we will present details about the current capacity for high-throughput crystal growth screening. We will also discuss innovations we are developing and opportunities for enhanced crystallization services that will further facilitate crystallization for biomolecular structure determination, including scale up and optimization, in situ diffraction experiments and enhanced imaging for crystal detection.

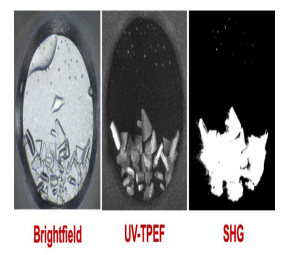

Figure 1

Acta Cryst. (2020). A76, a206 